\title{
Estudio del comportamiento viscoso de peloides termales
}

\author{
Carmen P. GÓMEZ ${ }^{(1)}, \mathrm{M}^{\mathrm{a}}$ Lourdes MOURELLE ${ }^{(1)}$, \\ Carlos MEDINA $^{(1)}, \mathrm{M}^{\text {a }}$ Perfecta SALGADO ${ }^{(1)}$, Sofía BAZ ${ }^{(1)}$, Marta ARRIBAS ${ }^{(2)}$ \\ ${ }^{(1)}$ Departamento de Física Aplicada. Facultad de Ciencias del Mar. Universidad de Vigo. \\ Campus As Lagoas Marcosende s/n, 36310 Vigo (España). \\ ${ }^{(2)}$ Servicio Médico. Balneario Isla de la Toja. Hotel Resort Hesperia Isla de la Toja. \\ 36991 O Grove, Pontevedra (España). \\ carmengomez@uvigo.es
}

Recibido: 19-01-11

Aceptado: 07-03-11

\section{Resumen}

A pesar de que se ha investigado ampliamente la composición y propiedades físicas de los peloides, existen pocos estudios con métodos analíticos relacionados con el manejo y la aplicabilidad sobre la piel, aspecto que es importante en la peloterapia. Existen estudios sobre la textura (adhesividad, cohesión, elasticidad) y sobre la afinidad sobre la piel (tensiometricprint), aunque escasos sobre la viscosidad y aspectos reológicos. La viscosidad es un parámetro importante para la aplicabilidad de un peloide, pero también para su caracterización termofísica.

La viscosidad de un peloide está estrechamente ligada a su contenido en agua. En las determinaciones de Ferrand e Yvon se estudiaron las viscosidades de pastas de caolinita y bentonita, observando que las mezclas de ambas espesan con el tiempo y no cumplen las leyes habituales de adición, sino que la viscosidad de las mezclas es menor que la de las pastas puras. Estudios experimentales llevados a cabo en el departamento de Física Aplicada de la Universidad de Vigo han sugerido asimismo que el tipo de agua mineral utilizada para la elaboración del peloide también posee gran importancia, ya que la viscosidad de estas mezclas varía según la mineralización del agua utilizada.

En este trabajo se han realizado diferentes determinaciones de la viscosidad de mezclas de una arcilla tipo bentonita con diferentes aguas: agua tridestilada, agua de mar y cuatro tipos de aguas mineromedicinales.

Palabras claves: Peloides termales, Peloterapia, Viscosidad

\section{Study of viscous behaviour in thermal peloids}

\begin{abstract}
Although there are many studies available on the composition and physical properties of peloids, there nevertheless are very few studies wherein analytical methods are related to handling and skin application-aspects which are important in peloid therapy. Literature is
\end{abstract}


also available on texture (adhesiveness, cohesion, elasticity) and on skin affinity (tensiometric print) but is less available on viscosity and rheological aspects. Viscosity is an important parameter not only for peloid application but also for its thermo-physical characterisation.

Peloid viscosity is closely linked to water content. Ferrand and Yvon studied viscosities in kaolinite and bentonite pastes. They noticed that mixtures of the two thickened over time and did not obey normal addition laws because the viscosity of their mixtures was observed to be lower than that of their individual pastes in pure form. Experimental studies carried out at the Department of Applied Physics, University of Vigo, have likewise suggested that the type of mineral water used for preparing the peloid is of great importance, since mixture viscosity varies according to mineralisation of water.

Several viscosity measurements were made using mixtures of a type of bentonite clay and varying proportions of different types of waters: tri-distilled water, seawater and four mineral-medicinal waters.

Key words: Thermal peloids, Pelotherapy, Viscosity

REFERENCIA NORMALIZADA

Gómez CP, Mourelle ML, Medina C, Salgado MP, Baz S, Arribas M. Estudio del comportamiento viscoso de peloides termales. (Study of viscous behaviour in thermal peloids). Anal Hidrol Med, 2011, vol. 4, 107-116

\section{INTRODUCCIÓN}

A pesar de que se ha investigado ampliamente la composición y propiedades físicas de los peloides, existen pocos estudios con métodos analíticos relacionados con el manejo y la aplicabilidad sobre la piel, aspecto que es importante en la peloterapia. Existen estudios sobre la textura (adhesividad, cohesión, elasticidad) ${ }^{1}$ y sobre la afinidad sobre la piel (tensiometricprint) ${ }^{2}$, aunque son escasos los relacionados con la viscosidad y los aspectos reológicos ${ }^{3}$. La viscosidad es un parámetro importante para la aplicabilidad de un peloide, pero también para su caracterización termofísica.

La viscosidad de un peloide está estrechamente ligada a su contenido en agua. En las determinaciones de Ferrand e Yvon ${ }^{4}$ se estudiaron las viscosidades de pastas de caolinita y bentonita, observando que las mezclas de ambas espesan con el tiempo y no cumplen las leyes habituales de adición, sino que la viscosidad de las mezclas es menor que la de las pastas puras. La viscosidad que alcanzan las pastas de arcillas con agua -en la preparación de peloides- está fuertemente influida por el contenido en agua de la mezcla, pero también por el tipo de material, ya que en las arcillas con mayor capacidad de hinchamiento (tipo esmectitas) se pueden conseguir pastas altamente viscosas con un elevado porcentaje de agua.

Estudios experimentales llevados a cabo en el departamento de Física Aplicada de la Universidad de Vigo han sugerido asimismo que el tipo de agua mineral 
utilizada para la elaboración del peloide también posee gran importancia, ya que la viscosidad de estas mezclas varía según la mineralización del agua ${ }^{5-6}$.

El objetivo de este trabajo es la realización de diferentes determinaciones de la viscosidad de mezclas de una arcilla tipo bentonita con diferentes aguas: agua tridestilada, agua de mar y agua mineromedicinal (MM) de los balnearios de Cuntis, Caldelas de Tui, Mondaríz e Isla de la Toja, utilizando diferentes porcentajes de agua.

\section{MATERIALES}

La bentonita es una arcilla compuesta esencialmente por minerales del grupo de las esmectitas. La utilizada en este estudio es una bentonita del tipo de las altamente hinchables (según clasificación Patterson y Murray). Se han llevado a cabo diferentes mezclas de esta bentonita con: agua de mar (Quinton), con agua tridestilada y con cuatro aguas mineromedicinales de balnearios de la CCAA de Galicia que poseen aguas con diferente mineralización.

Las características de las muestras utilizadas se muestran en la tabla 1.

Tabla 1 - Características de las aguas utilizadas

\begin{tabular}{|c|c|c|c|}
\hline Agua MM & $\begin{array}{c}\text { Residuo seco } \\
\text { a } 110^{\circ} \mathrm{C}(\mathrm{mg} / \mathrm{l})\end{array}$ & $\begin{array}{l}\text { Conductividad } \\
(\mathrm{mS} / \mathrm{cm}) \text { a } 25^{\circ} \mathrm{C}\end{array}$ & $\begin{array}{c}\text { Clasificación } \\
\text { (Maraver, 2004) }\end{array}$ \\
\hline $\begin{array}{c}\text { Balneario } \\
\text { Caldelas de Tui }\end{array}$ & $1.461,98$ & 1,097 & $\begin{array}{l}\text { Iones predomi- } \\
\text { nantes cloruro, } \\
\text { sodio }\end{array}$ \\
\hline $\begin{array}{l}\text { Balneario de } \\
\text { Cuntis }\end{array}$ & $1.636,66$ & 0,483 & Sulfurada \\
\hline $\begin{array}{c}\text { Balneario La } \\
\text { Toja }\end{array}$ & $31.164,24$ & 44,90 & $\begin{array}{l}\text { Clorurada, sódi- } \\
\text { ca, ferruginosa, } \\
\text { radiactiva }\end{array}$ \\
\hline $\begin{array}{l}\text { Balneario } \\
\text { Mondariz } \\
\text { (Gándara) }\end{array}$ & 2.971,77 & 1,173 & $\begin{array}{l}\text { Carbogaseosa, } \\
\text { radiactiva }\end{array}$ \\
\hline $\begin{array}{l}\text { Agua de mar } \\
\text { Quinton }\end{array}$ & $40.203,03$ & 50,90 & \\
\hline
\end{tabular}




\section{MÉTODOS}

Para la determinación de las viscosidades se ha utilizado el viscosímetro Visco Easy 1.0 SCHOTT (figura 1). Este viscosímetro funciona por el principio de rotación de un cilindro o bien de un disco sumergido en el material que debe ser probado, midiendo la fuerza de torsión necesaria para superar la resistencia viscosa de la rotación. El cilindro o disco (husillo giratorio), está acoplado con un muelle al árbol motor que gira a una velocidad determinada. El ángulo de desviación del eje se mide electrónicamente dando la medida de la fuerza de torsión.

\section{Figura 1 - Viscosímetro Visco Easy 1.0 Schott}

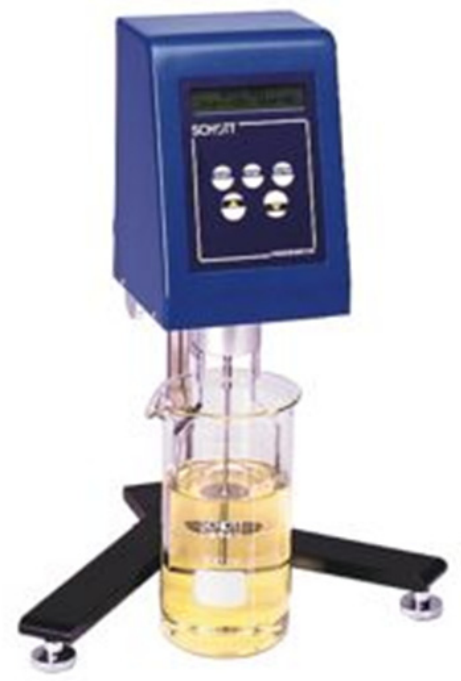

Los cálculos realizados dentro del viscosímetro a partir de las medidas de torsión, de la velocidad del eje y de sus características, dan una lectura directa de la viscosidad en mPa.s.

El equipo dispone de varios tipos de husillos y de una extensa gama de velocidades, proporcionando así una gran capacidad de medición de la viscosidad. Para cualquier fluido de viscosidad determinada, la resistencia al avance crece proporcionalmente a la velocidad de rotación del husillo o al tamaño del mismo. El viscosímetro está construido para tener en cuenta la velocidad seleccionada y el tipo de husillo escogido para dar los resultados en mPa.s. Las combinaciones de husillos, permiten elegir una escala óptima para cualquier medición, dentro de la gama del aparato. 
Los cambios de rango pueden realizarse utilizando el mismo husillo a diferentes velocidades.

\section{RESULTADOS Y DISCUSIÓN}

La textura adecuada de las mezclas de la bentonita sódica con las diferentes aguas mineromedicinales, de mar y tridestilada, se alcanza con diferentes proporciones de arcilla (entre un 16\% para el agua tridestilada y un $34 \%$ para el agua mineromedicinal del Balneario de La Toja).

En la tabla 2 se muestran los valores de las viscosidades a $25^{\circ} \mathrm{C}$ y $35^{\circ} \mathrm{C}$ de los porcentajes idóneos de las mezclas.

Tabla 2 - Viscosidades de las mezclas en función del contenido de arcilla y la temperatura

\begin{tabular}{|cccc|}
\hline Tipo de aguas & \% arcilla & $\begin{array}{c}\text { Viscosidad (Pa.s) } \\
\mathbf{a ~ 2 5}^{\mathbf{0}} \mathbf{C}\end{array}$ & $\begin{array}{c}\text { Viscosidad (Pa.s) } \\
\mathbf{a ~ 3 5}^{\mathbf{0}} \mathbf{C}\end{array}$ \\
\hline $\begin{array}{c}\text { Balneario } \\
\text { Cuntis }\end{array}$ & 22 & 350 & 335 \\
$\begin{array}{c}\text { Balneario } \\
\text { La Toja }\end{array}$ & 34 & 79 & 76 \\
$\begin{array}{c}\text { Balneario Caldelas } \\
\text { de Tui }\end{array}$ & 20 & 214 & 198 \\
$\begin{array}{c}\text { Baln. Mondariz } \\
\text { (Gándara) }\end{array}$ & 18 & 147 & 146 \\
$\begin{array}{c}\text { Agua de mar } \\
\text { Quinton } \\
\text { Agua }\end{array}$ & 33 & 38 & 35 \\
tridestilada & 16 & 73 & 73 \\
\hline
\end{tabular}

En las figuras 2 a 7 se puede observar que la viscosidad disminuye al aumentar la temperatura y que, partiendo de una misma arcilla, con diferentes aguas mineromedicinales, el peloide genera una estructura de pasta con diferentes concentraciones y varía con el tipo de agua y con la mineralización de la misma. 
Figura 2 - Viscosidades de mezclas del agua mineromedicinal del Balneario de Cuntis con bentonita

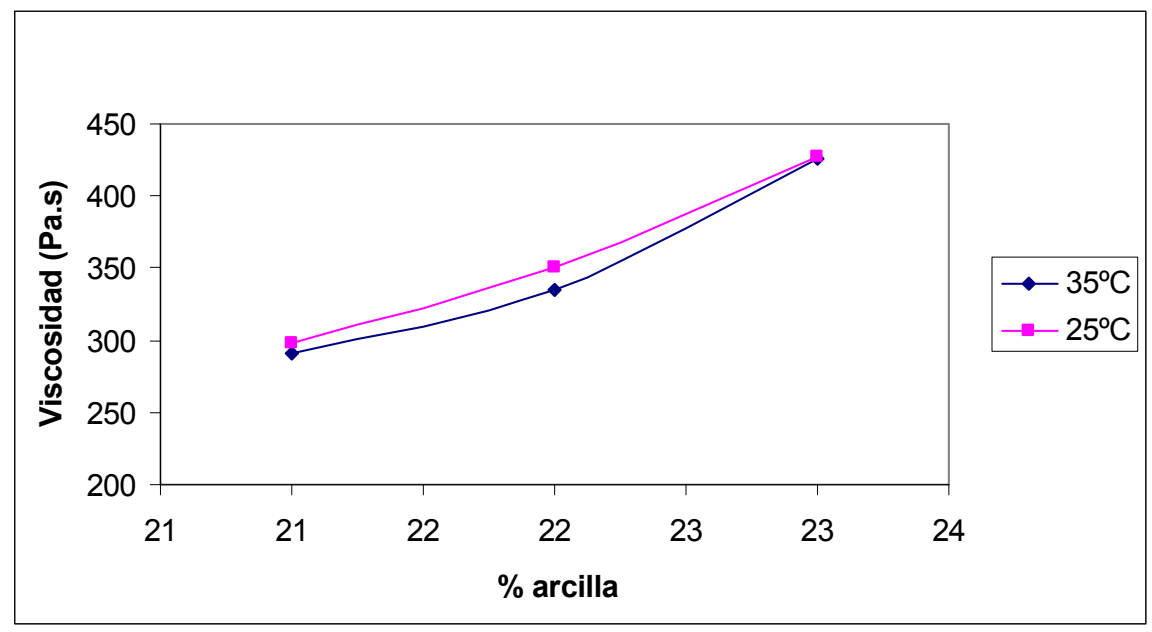

Figura 3 - Viscosidades de mezclas del agua mineromedicinal del Balneario de La Toja con bentonita

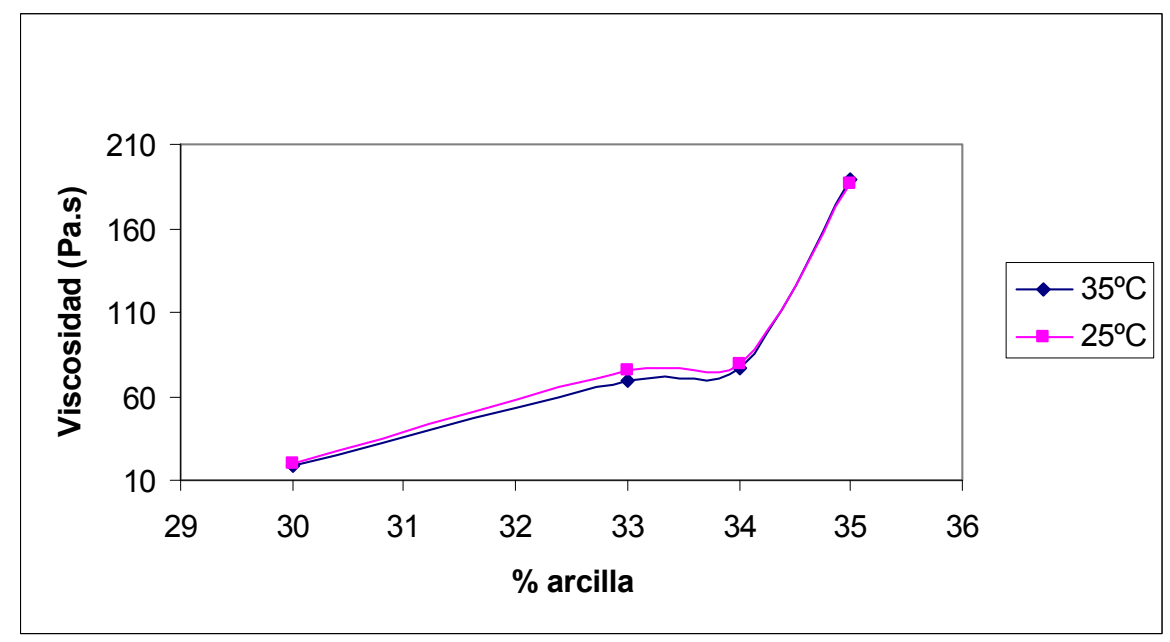


Figura 4 - Viscosidades de mezclas del agua mineromedicinal del Balneario de Caldelas de Tui con bentonita

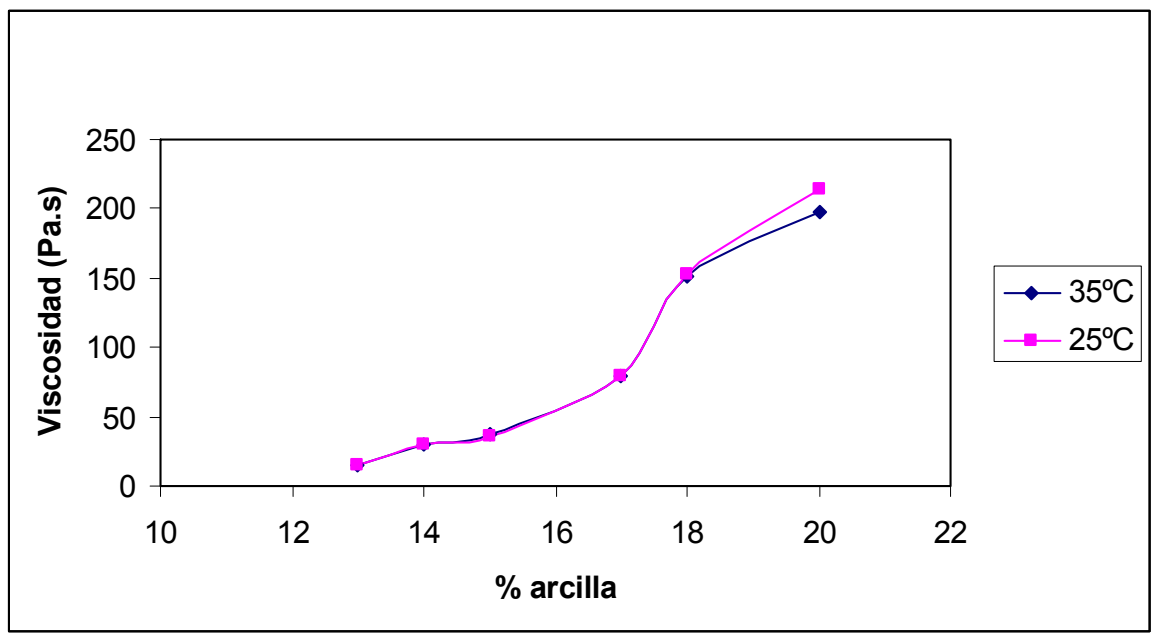

Figura 5 - Viscosidades de mezclas del agua mineromedicinal del Balneario de Mondaríz con bentonita

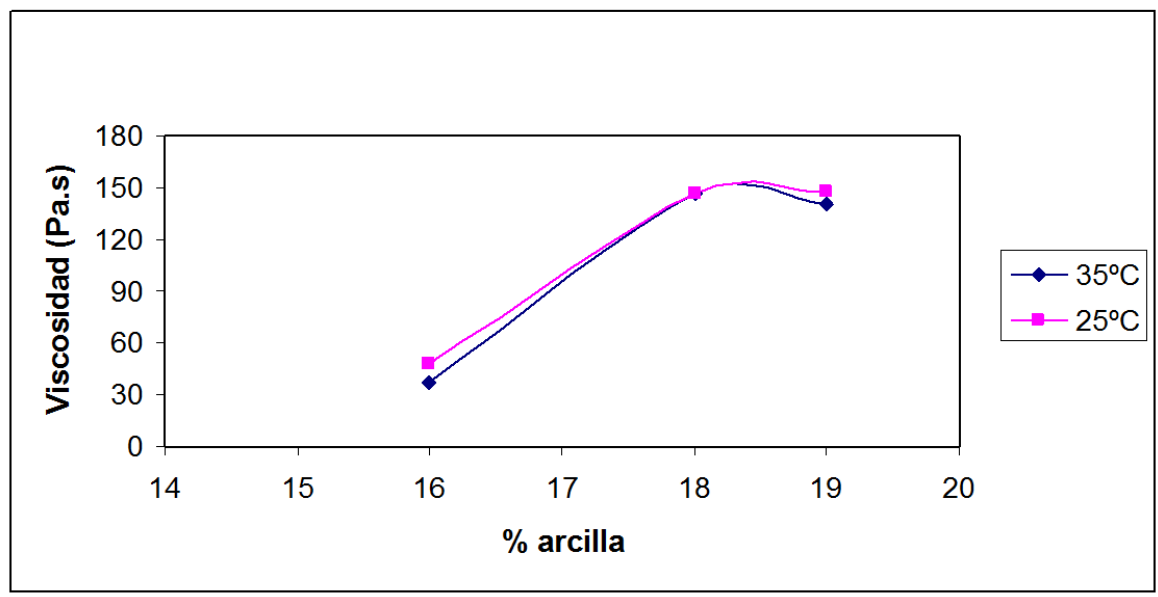


Figura 6 - Viscosidades de mezclas del agua de mar Quinton con bentonita

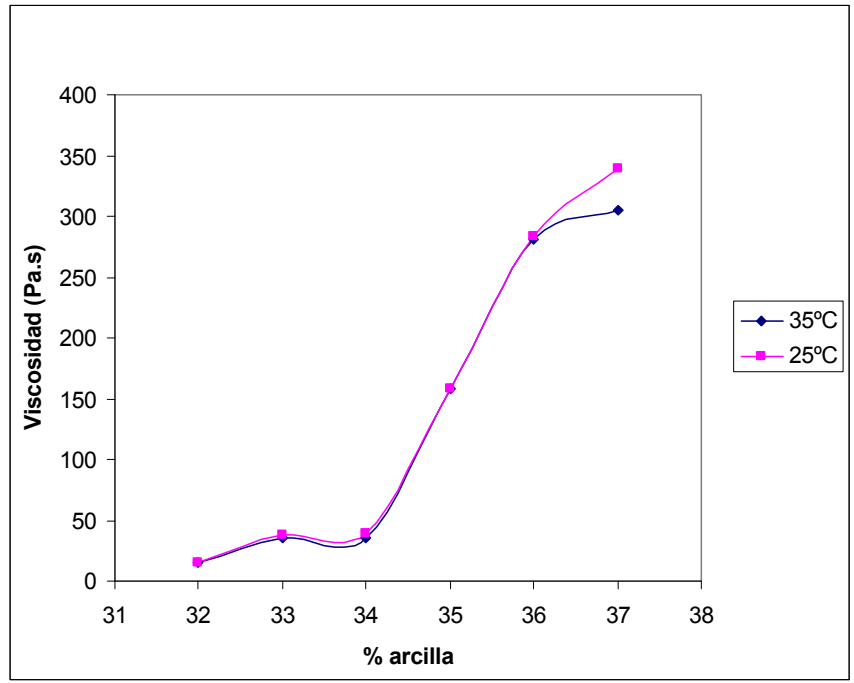

Figura 7 - Viscosidades de mezclas del agua tridestilada con bentonita

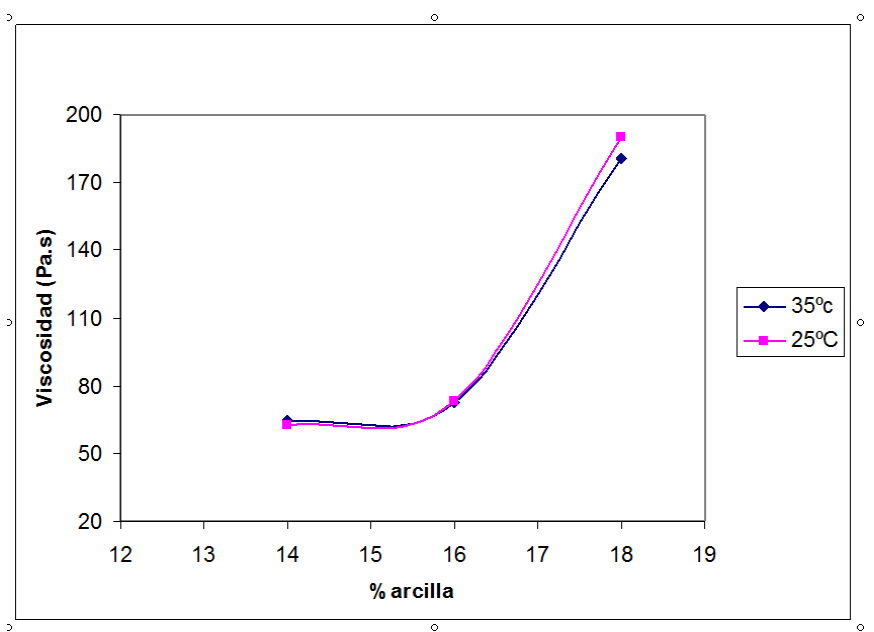




\section{CONCLUSIONES}

Los resultados muestran que las propiedades reológicas de los peloides son altamente dependientes de la mineralización del agua utilizada.

Para la preparación de un peloide es importante tener en cuenta el porcentaje y el tipo de agua utilizada para alcanzar una textura apropiada. Además de tener en cuenta las propiedades termofísicas (calor específico, conductividad térmica, densidad, etc.) es preciso también prestar atención a las propiedades reológicas para obtener un producto con una buena consistencia y fácil de extender y, por lo tanto, con una viscosidad intermedia.

\section{Agradecimientos}

Esta investigación ha sido financiada por los proyectos de $\mathrm{I}+\mathrm{D}+\mathrm{i}$ de la Xunta de Galicia PGIDT07PXIB310190PR y 10PXIB310153PR y por el proyecto TERMARED (Sudoe-Interreg IV).

Agradecemos a los Laboratorios Quinton y a los balnearios de Cuntis, Caldelas de Tui, Mondaríz e Isla de la Toja su contribución facilitando las muestras de agua estudiadas.

Agradecemos también la asistencia técnica del Sr. Jorge Millos y la Sra. Estefanía López del CACTI (Centro de Apoyo Científico y Tecnológico a la Investigación, Universidad de Vigo).

\section{BIBLIOGRAFIA}

1. Armijo F, Maraver F. Granulometría y textura de los peloides españoles. Anal Hidrol Med 2006; 1: 79-96.

2. Rossi D, Dal Bosco C, Jobstraibizer PG, Setti M, Veniale F, Bettero A. Thermal muds evaluation by tensiometric versus skin modeling (TVS modeling $\left.{ }^{\circledR}\right)$. En: Legido JL, Mourelle ML (eds). Investigaciones en el ámbito Iberoamericano sobre peloides termales. Vigo: Universidad de Vigo, 2007: 151-159.

3. Beer AM, Grozeva A, Sagorchev P, Lukanov J. Comparative study of the thermal properties of mud and peat solutions applied in clinical practice. Biomedizinische Technik 2003; 48: 301-305.

4. Ferrand T, Yvon J. Thermal properties of clay pastes for pelotherapy. App Clay Sci 1991; 6: 21-38.

5. Mourelle ML, Gómez CP, Medina C, Legido JL. Características de peloides extemporáneos elaborados con distintas aguas mineromedicinales. Bol Soc Esp Hidrol Med 2010; 25(1): 72-73. 
6. Mourelle ML, Gómez-Pardo C, Medina C; Legido, JL, Navia P, Romaní L, Meijide R. Thermophysical and rheological caracterization of peloids from several mineral waters. Press Therm Climat 2010; 147(1): 103. 